Pour la première fois en Suisse, la question du «number needed to treat»

a fait l'objet d'une procédure judiciaire

\title{
Défenestration et Tribunal fédéral
}

Hanspeter Kuhn, avocat et secrétaire général adjoint

Toutes les citations du présent article ont été traduites par la FMH.
Dans son arrêt concernant une «défenestration» de février 2006, le Tribunal fédéral estime que l'hôpital aurait dû ordonner une garde de nuit personnelle pour un patient opéré du cœur, d'autant plus elle n'aurait coûté que Fr. 1680.pour une semaine. Dans ce contexte, le Tribunal fédéral n'a pas vu la question du nombre de sujets à traiter («number needed to treat»). Le tribunal administratif cantonal l'avait analysée et était parvenu à des coûts marginaux d'1,7 milliard de francs. Autrement dit: la sécurité exigée par le Tribunal fédéral ne serait définitivement plus finançable. Reste à espérer qu'il corrigera sa jurisprudence à la prochaine occasion.

En l'espèce, un point qui n'a pas vraiment été examiné dans le cadre du litige est celui de l'information. Si les hôpitaux n'appliquent pas - à juste titre selon moi - l'arrêt du Tribunal fédéral sur une large échelle, l'information devient importante. La question est évidente: le patient ou ses proches veulent-ils financer eux-mêmes une garde personnelle à l'hôpital quand la collectivité ne peut pas la payer en raison de coûts marginaux trop élevés?

Une défenestration antérieure avait déclenché une guerre européenne. Le cas présent n'occupe que la Suisse. Il constitue néanmoins une première pour notre petit pays, car le Tribunal fédéral a dû se prononcer pour la première fois sur un traitement impliquant la question du nombre de sujets à traiter et celle des coûts marginaux d'une sécurité supplémentaire en médecine. Mais procédons par ordre:

\section{Le cas}

Un patient se trouvait dans un hôpital universitaire suisse où il avait été opéré du cœur. La fenêtre de sa chambre était fermée de manière sécurisée parce que, depuis quelque temps, il présentait des troubles mentaux dès le soir venu. Le rapport médical cité dans l'arrêt du tribunal a la teneur suivante: «A 00h15, le médecin a constaté que le patient était calme, qu'il répondait correctement et qu'il ne présentait aucun trouble neurologique.» L'invraisemblable s'est produit vingt minutes plus tard: «A 00h35, il a sauté du balcon de la chambre voisine [mise en italique par l'auteur] pour décéder de sa chute.» Destin ou faute de traitement?

\section{Le premier arrêt du tribunal cantonal}

Le tribunal administratif cantonal s'était prononcé ainsi en première instance:

«Le fait de renoncer à une garde personnelle qui, au $\mathrm{vu}$ du déroulement inhabituel des événements, avait été laissé à l'appréciation des responsables, ne doit pas être considéré comme un manquement au devoir de diligence.» [1]

\section{Le premier arrêt du Tribunal fédéral (2004) [2]}

Le Tribunal fédéral a invalidé le jugement du tribunal cantonal. Cette annulation était compréhensible, car la première instance ne s'était pas prononcée elle-même sur le bien-fondé d'une garde mais avait délégué de facto cette question aux experts.

\section{Le deuxième arrêt du tribunal cantonal}

L'hôpital concerné n'avait pas ordonné moins de gardes personnelles, en moyenne, que d'autres hôpitaux universitaires comparables. Il restait à savoir si l'hôpital aurait dû commander, aussi pour ce patient, une garde personnelle dans le cadre de la diligence que l'on peut raisonnablement attendre afin de l'empêcher de se jeter de la fenêtre de la chambre voisine.

Le 25 juillet 2005, le tribunal administratif cantonal s'est prononcé comme suit: la fenêtre de la chambre de ce patient était fermée de manière sécurisée. Selon les experts, une défenestration dans la chambre voisine ne s'était encore jamais produite de par le monde. Si l'on veut une sécurité maximale, on doit organiser, après une opération cardiaque, une garde personnelle pendant une semaine pour chaque patient confus, donc prévoir une charge supplémentaire de Fr. 1680.- par patient.

L'utilité marginale en serait faible car la probabilité d'une défenestration dans la chambre voisine s'élève à une contre un million. Les coûts marginaux deviennent ainsi astronomiquement élevés:

\footnotetext{
«Si l'on met cette réduction du risque en rapport avec les coûts d'une garde personnelle pendant une semaine (Fr. 1680.-; cf. ci-avant arrêt 5.4.1), laquelle aurait pu éviter l'événement fatal (ci-avant arrêt 5.4.3), les coûts qui en résultent par vie humaine sauvée s'élèvent au minimum à env. 1,7 milliard de francs, ou, calculés d'après l'espérance de vie moyenne d'env.
} 
11,5 ans du patient âgé de 75 ans à l'époque, (Stauffer/Schaetzle, Barwerttafeln, 5e édition 2001, tableau 42), à 150 millions de francs au minimum par année de vie humaine sauvée. [...] Même si l'on changeait clairement différents paramètres de ce calcul en les faisant pencher de manière irréaliste en faveur des plaignantes, une telle mesure ne pourrait plus être considérée comme acceptable sur le plan financier.» (arrêt 5.4.6)

Le tribunal administratif cantonal compare ces coûts marginaux à ceux réputés raisonnables en santé publique et dans la discussion générale sur les risques:

«En santé publique, on admet des coûts jusqu'à Fr. 300 000.-env. par QALY (quality-adjusted life year ou «année de vie ajustée sur la qualité») même dans le cadre de méthodes thérapeutiques compliquées (cf. Jürg H. Sommer, Muddling Through Elegantly: Rationierung im Gesundheitswesen, 2001, p. 69 ss.). Dans la discussion générale sur les risques, on admet des coûts marginaux de 20 millions de francs au maximum par vie humaine sauvée ou de Fr. 500 000.- au maximum par année de vie sauvée (Hansjörg Seiler, p. 153 s. [3]).»

Le tribunal administratif cantonal estime donc en conclusion que l'hôpital universitaire ne pouvait ni ne devait organiser une garde personnelle pour ce patient aux frais des assurés et des contribuables [4].

Dans le beau récit publié en 2003 par Luzi Dubs [7], le Dr Klöti, médecin de campagne, résout élégamment la question sur laquelle a trébuché le Tribunal fédéral:

\section{«Visite du représentant Ledergerber chez le docteur Klöti}

Travaillant depuis quinze ans comme médecin de campagne, le Dr Pius Klöti est satisfait de son activité et reçoit chaque année un représentant en produits pharmaceutiques qui l'informe sur les médicaments les plus récents. [...] Tout en étalant sur la table ses premiers échantillons, Ledergerber mentionne fièrement le prix mensuel avantageux de Fr. 78.- pour un traitement.

Klöti paraît plus pensif qu'à l'ordinaire et réfléchit plus longuement que de coutume à l'étude qu'on lui soumet. A l'endroit où sont présentés les chiffres concrets en matière d'incidences, il s'arrête: une prise de médicaments pendant dix ans réduit le taux de fractures du col du fémur de 6 à $4 \%$. Un calcul rapide de la réduction absolue du risque l'amène à un «nombre de sujets à traiter» de 50. II devrait par conséquent traiter 50 femmes avec CONTRAFRAKT-PLUS pendant dix ans pour éviter une seule fracture du col du fémur. En partant d'un prix annuel de 936 francs par personne, il faut donc compter Fr. 468 000.- pour atteindre cet objectif. Certes, en mentionnant une réduction relative du risque de $33 \%$, Ledergerber $n^{\prime}$ a rien dit qui ne soit vrai. Mais, cette fois-ci, le Dr Klöti ne s'y est pas laissé prendre.»

Un livre à lire

L'ouvrage de Gerg Gigerenzer «Das Einmaleins der Skepsis» [8, 9] a obtenu en 2002 en Allemagne le prix du meilleur livre scientifique de I'année. D'une écriture fraîche et légère, il constitue une excellente lecture de chevet pour les médecins et les juristes.

\section{Commentaire}

Cette approche méthodique est convaincante. Lors de décisions concernant un traitement, la médecine et le droit doivent, en effet, soupeser l'utilité marginale et les coûts marginaux.

Compte tenu de l'événement concret faisant l'objet du procès, le tribunal administratif n'a examiné l'utilité marginale et les coûts marginaux qu'en regard de la défenestration dans la chambre voisine. C'était probablement quelque peu réducteur et la question demeure finalement sans réponse: par-dessus tout, à combien s'élèverait l'utilité marginale si l'indication pour des gardes personnelles était plus souple, et quel serait le montant des coûts marginaux qui en résulteraient?

\section{Le deuxième arrêt du tribunal fédéral (2006) [5]}

Certes, le Tribunal fédéral prend acte de la discussion du tribunal administratif cantonal sur l'utilité marginale et les coûts marginaux:

\begin{abstract}
«D'après les chiffres mentionnés (ci-avant arrêt 4.1), les coûts par patient confus s'élèvent à Fr. 1680.et pour l'ensemble des cas, entre Fr. 369600 .- et Fr. 655 200.- par année [pour cet hôpital (remarque de l'auteur)]. Ces coûts apparaissent sans autres financièrement supportables, en particulier lorsqu'on les compare aux coûts, bien plus élevés, des opérations effectués sur ce genre de patients. D'ailleurs, le résultat est encore plus net lorsqu'on reprend les considérations économiques émises par le tribunal administratif. Ce dernier estime les coûts marginaux à vingt millions de francs au maximum par vie humaine sauvée et de Fr. 300 000.- à Fr. 500 000.- par année de vie sauvée (arrêt 5.2 p. 20). Pour Monsieur H._ qui bénéficiait d'une espérance de vie moyenne de 11,5 ans au moment de l'opération (jugement attaqué, arrêt 5.4.6), les coûts marginaux se seraient ainsi élevés entre Fr. 3450000 .- et Fr. 5750 000.- (arrêt 4.3.).»
\end{abstract}

Toutefois, le Tribunal fédéral montre immédiatement qu'il n'a pas encore compris cette logique car il poursuit:

«Comparée à ces montants, la dépense exigée de Fr. 1680.- pour une garde personnelle d'une semaine est extrêmement basse, raison pour laquelle il est possible de reconnaître sans autres l'applicabilité financière d'une telle mesure, même sous cet angle.»

\section{Commentaire}

\section{L'arrêt est illogique et non finançable}

Il apparaît que les deux tribunaux ont «tiré l'un sur l'autre» dans le cas d'espèce, ce qui complique l'évaluation des conséquences que l'on peut en tirer.

Néanmoins, une chose est claire, à mon avis: l'arrêt du Tribunal fédéral n'est pas convaincant. Le Tribunal fédéral n'a apparemment pas encore 
compris la question du nombre de sujets à traiter. Autrement dit, il serait médicalement et juridiquement faux d'ordonner une garde personnelle pour chaque patient cardiaque confus. Le tribunal administratif cantonal a relevé à juste titre ceci:

«Il serait en effet inconciliable avec le principe de l'égalité (art. 8, 1er al. de la Constitution fédérale) d'engager dans quelques cas des dépenses très élevées pour continuer à réduire des risques minimes, alors qu'aucune mesure même nettement moins onéreuse n'est prise dans d'autres cas. Cette remarque est également valable pour les risques en santé publique: il ne s'agit pas non plus ici de prendre des mesures de sécurité très dispendieuses uniquement pour certains patients ou catégories de patients et pas pour d'autres, car toutes les personnes ont un droit égal à des traitements ou autres mesures (cf. Brigitte Pfiffner Rauber, Das Recht auf Krankheitsbehandlung und Pflege, 2003, p. 50 ss., 223 ss.). Il n'est donc possible d'accepter financièrement la réalisation de mesures qu'aussi longtemps que les charges qui en découlent, précisément lorsqu'elles deviennent générales, restent dans un cadre supportable pour la politique de la santé ou les fonds publics.»

Il n'y a rien à ajouter à ces considérations.

\section{Information au patient sur la possibilité de financer lui-même des mesures trop chères?}

Les traitements effectués aux frais des assurés/ contribuables doivent être efficaces, adéquats et économiques. En revanche, rien n'interdit au patient de payer lui-même des traitements utiles mais non économiques. Tant qu'une utilité médicale marginale subsiste (donc tant que la mesure supplémentaire aide davantage qu'elle ne nuit), la question se pose de savoir si le médecin ne devrait pas laisser au patient ou à ses proches le soin de décider: veulent-ils prendre en charge eux-mêmes cette mesure additionnelle malgré un coût marginal élevé, voire astronomique dans la situation qui nous occupe, ou, dans un autre cas par exemple, payer eux-mêmes un médicament qui n'est pas remboursé par les caissesmaladies [6]?

Une garde personnelle effectuée par les proches du patient n'est en revanche pas une solution envisageable. Tous les médecins hospitaliers que j'ai contactés à ce propos ont eu la même réaction spontanée: «Un parent du malade à l'hôpital pendant la nuit, c'est un patient de plus à prendre en charge.» On en reviendrait par là indirectement aux coûts supplémentaires visant à assurer une sécurité additionnelle, coûts qu'il n'est plus possible de faire assumer aux assurés/ contribuables.

\section{Références}

1 Décision cantonale reprise dans l'arrêt du Tribunal fédéral de 2004.

2 Arrêt du 19 octobre 2004, 4P.92/2004.

3 Le tribunal se réfère ici à: Seiler H. Risikobasiertes Recht. Risk-Based-Regulation - ein taugliches Konzept für das Sicherheitsrecht? Fond national suisse, projet no 1113-52163.97. Bern: Stämpfli; 2000.

4 «Dans l'ensemble, il ressort de ces considérations qu'étant donné la très faible probabilité de décès qui aurait pu être évitée avec une garde personnelle, cette mesure n'était financièrement pas applicable et que sa non-application ne contrevenait pas au devoir de diligence.» Arrêt 5.6.

5 Arrêt numéro 4P.244/2005.

6 Jefford M, et al. Medical Paternalism and expensive unsubsidised drugs. BMJ 2005;331:1075-80.

7 Dubs L. Offensive für nutzenorientierte Medizin in Winterthur. Schweiz Ärztezeitung 2003; 84(35):1790-1.

8 Gigerenzer G. Das Einmaleins der Skepsis. Über den richtigen Umgang mit Zahlen und Risiken. $2^{e}$ édition. Berlin: Berliner Taschenbuch Verlag; 2005.

9 Gigerenzer G. Calculated risks: How to know when numbers deceive you. New York: Simon \& Schuster (UK edition: Penguin Books); 2002. 